\title{
MONSTER v1.1: a tool to extract and search for RNA non-branching structures
}

\author{
Giulia Fiscon ${ }^{1,2^{*}}$, Paola Paci', Giulio lannello ${ }^{1,3}$ \\ From Eleventh Annual Meeting of the Bioinformatics Italian Society Meeting \\ Rome, Italy. 26-28 February 2014
}

\begin{abstract}
Background: Detection of RNA structure similarities is still one of the major computational problems in the discovery of RNA functions. A case in point is the study of the new appreciated long non-coding RNAs (IncRNAs), emerging as new players involved in many cellular processes and molecular interactions. Among several mechanisms of action, some IncRNAs show specific substructures that are likely to be instrumental for their functioning. For instance, it has been reported in literature that some IncRNAs have a guiding or scaffolding role by binding chromatin-modifying protein complexes. Thus, a functionally characterized IncRNA (reference) can be used to infer the function of others that are functionally unknown (target), based on shared structural motifs.

Methods: In our previous work we presented a tool, MONSTER v1.0, able to identify structural motifs shared between two full-length RNAs. Our procedure is mainly composed of two ad-hoc developed algorithms: nbRSSP_extractor for characterizing the folding of an RNA sequence by means of a sequence-structure descriptor (i.e., an array of non-overlapping substructures located on the RNA sequence and coded by dot-bracket notation); and SSD_finder, to enable an effective search engine for groups of matches (i.e., chains) common to the reference and target RNA based on a dynamic programming approach with a new score function. Here, we present an updated version of the previous one (MONSTER v1.1) accounting for the peculiar feature of IncRNAs that are not expected to have a unique fold, but appear to fluctuate among a large number of equally-stable folds. In particular, we improved our SSD_finder algorithm in order to take into account all the alternative equally-stable structures.

Results: We present an application of MONSTER v1.1 on lincRNAs, which are a specific class of IncRNAs located in genomic regions which do not overlap protein-coding genes. In particular, we provide reliable predictions of the shared chains between HOTAIR, ANRIL and COLDAIR. The latter are lincRNAs which interact with the same protein complexes of the Polycomb group and hence they are expected to share structural motifs.

Software availability: the software package is provided as additional file 1 ("archive_updated.zip").
\end{abstract}

\section{Background}

The last years have been the scene of increasing interest in long non-coding RNAs (lncRNAs), a large and heterogonous class of RNAs not translated into proteins longer than 200 nucleotides [1-4]. These novel genes appear often deregulated in cancer $[5,6]$ and are emerging as new players of transcriptional and post-transcriptional regulation [7-9]. However, only a small subset of them

\footnotetext{
* Correspondence: fiscon@dis.uniroma1.it

"Institute for System Analysis and Computer Science "Antonio Ruberti" (IASI), National Research Council (CNR), Via dei Taurini 19, 00185 Rome, Italy Full list of author information is available at the end of the article
}

has already been functionally characterized. Among them, there is a specific sub-group of lncRNAs, called lincRNAs (large intergenic non-coding RNAs), that reside in genomic regions which do not overlap protein-coding genes [10-14]. This positive feature in light of experimental manipulation favored them in pioneer functional studies [15]. Indeed, some lincRNAs (e.g., HOTAIR, ANRIL, and COLDAIR) constitute exemplar lncRNAs whose function can be related to their structure (see Table 1). It has been suggested as all of them interact with chromatin-remodeling complexes and specifically with the Polycomb Repressive Complex 2 [16-18]. Therefore, they 
Table 1. Summary of our three case-of-study lincRNAs

\begin{tabular}{lll}
\hline lincRNA & Description & Function \\
\hline HOTAIR & $\begin{array}{l}\text { HOX intergenic antisense transcript localized in the } \\
\text { nucleus }\end{array}$ & $\begin{array}{l}\text { Epigenetically silences gene expression of many loci, through the recruitment } \\
\text { of chromatin-modifying complexes, such as PRC2, REST and CoREST. Its } \\
\text { expression is increased in tumor cells and may have an active role in the } \\
\text { epigenetic modulation of cancer and in mediation of the cells [11,10,39]. } \\
\text { COLDAIR }\end{array}$ \\
$\begin{array}{llll}\text { Transcribed in response to biological significant events, } \\
\text { such as a signal of cold environmental temperature }\end{array}$ & $\begin{array}{l}\text { Binding the protein complex PCR2 and performing the role of a guide for the } \\
\text { latter, determines the epigenetic repression of FLC gene (locus floral C) [40,10]. } \\
\text { Antisense non coding transcript in the INK4 locus }\end{array}$ & $\begin{array}{l}\text { Molecular scaffold for chromatin-modifying complexes PCR1 and PCR2, allows } \\
\text { to dynamically modulating transcriptional activity [41,10]. It is implicated in a } \\
\text { range of complex diseases including cancer and coronary heart diseases. }\end{array}$ \\
\hline
\end{tabular}

can be expected to share structural motifs. Thus, a functionally characterized lncRNA (here called reference) can be used to infer the function of other lncRNAs that are functionally unknown (here called target), based on shared structural motifs. This implies to assign a structure to the reference RNA and to look for structural similarities with a target RNA.

Although there are several tools performing the RNA secondary structure prediction, as well as detecting RNA structural similarities, they are not immediately suitable to deal with lincRNAs for two main reasons. First, large part of them are unable to efficiently treat long nucleotide sequences; second, most of the existing tools requires multiple sequence alignments, which are generally not available for lncRNAs.

In fact, an RNA secondary structure can be predicted using two main approaches: single-sequence [19] and comparative analysis [20]. The first class of methods performs prediction starting from single sequences using techniques that includes Free-Energy Minimization (e.g., Mfold [21] and RNAfold [22]) and machine learning (e.g., ContextFold [23]); while the second one makes predictions for sequence families, exploiting multiple sequence alignments to predict a consensus structure shared by all (or most) sequences in the alignment (e.g., RNAalifold [24], Dynalign [25], Carnac [26]). However, comparative analysis requires a large multi-alignment of available homologous sequences, which presently makes it not eligible approach for long and not conserved sequences of RNAs. Therefore, we focus on single-sequence methods and among them on the tools using the thermodynamic models, which result faster and do not require multiple alignments $[27,28]$. Thermodynamic methods rely on evaluation of the stability of a structure either quantifying free energy values [21], or assigning to each structure a probability according to the Boltzmann factor [29,30], or predicting the structure with the highest sum of base paring probability (Maximum Expected Accuracy structure [31]). Moreover, some of them can perform a global folding (e.g., Fold of RNAstructure [32] and RNAfold of Vienna RNA package), while other ones favor a local folding (i.e., RNALfold [33]). Since we deal with RNA of long sequences, we prefer the local folding software than the global ones. Indeed, a local folding that takes into account short-range pairs is less computational onerous and mostly more accurate than the global one [34].

For what concerns the search for structural similarities between two lncRNAs, up-to-date Structator [35] stands out as a computationally efficient tool to deal with long sequences. However, it does not adequately reward the rightness of the structures relative position in both the reference and the target.

In our previous work [36], we proposed a novel tool (MONSTER v1.0) that enables to detect structural motifs shared between two RNAs, taking as input only the RNA sequences independently from their nucleotides length, long as well as small. Following [35], we characterized the folding of an RNA sequence by means of a Secondary Structure Descriptor (SSD), i.e., an array of non-overlapping substructures, called Non Branching Structures (NBSs), and located on the RNA sequence. MONSTER v1.0 has been composed of two main core modules: (i) nbRSSP_extractor, to assign a unique structure to the reference RNA encoded by a set of NBSs; and (ii) SSD_finder, to detect structural motifs that the given target RNA has in common with the reference through an appropriate score function that takes into account the relative position among their NBSs.

On the other hand, lncRNAs as RNA molecules are not expected to have a unique fold, but appear to fluctuate among a large number of equally-stable folds [37]. Here, we present an updated version MONSTER v1.1, where we improved our SSD_finder algorithm in order to take into account all the alternative equally-stable structures. The improved version of $S S D_{-}$finder includes its old version as a particular case. Hereafter, we call $S S D \_$finder its improved version.

We thoroughly discuss the complexity of the $S S D_{-}$finder and we evaluate its robustness in identifying members of some RNA families using Rfam 11.0 freely available dataset. Finally, we apply our pipeline to the three well functionally characterized abovementioned lincRNAs (i.e., HOTAIR, ANRIL, and COLDAIR). 


\section{Methods}

The pipeline implemented in MONSTER and sketched in Figure 1 has been applied to lincRNAs here. It can be summarized in the following steps:

1. Selection of one lincRNA as reference (e.g., HOTAIR/ANRIL/COLDAIR) to compare with the other selected lincRNAs used as targets.

2. Prediction of the reference secondary structure (using RNALfold).

3. Extraction of the NBSs of the reference (using nbRSSP_extractor).

4. Encoding of reference NBSs into suitable SSD (using nbRSSP_extractor).

5. Searching for matches between the reference NBSs and target sequences (using Structator).

6. Filtering out matches with low probability to fold into the corresponding local NBS of the reference (using our match_filter).

7. Detecting putative set of motifs shared between reference and target (using SSD_finder). nbRSSP_extractor makes use of the local folding tool RNALfold [33] from the Vienna RNA Package to obtain the folding predictions of the reference. Then, based on an ad-hoc selection, it extracts and encodes the more stable NBSs in a SSD (Figure 2).

RNALfold is a Minimum Free Energy-based predictor that returns the locally stable secondary structures of an RNA sequence according to a given parameter $L$ that represents the maximum allowed distance between base-pairs. Additionally, it computes for each local structure its free energy as well as the starting position in the sequence. The output list is composed of all the possible local structures which are predicted and may overlap (i.e., more predictions correspond to an identical piece of sequence). The usage of RNALfold stems from its specific features: (i) it does not require homologous sequences that would be not available for lncRNAs, and (ii) it performs a local folding with lower computational costs than the other ones. In greater details, starting from a RNA sequence (Figure 2a), the predicted RNA secondary structure (Figure 2b) is broken down into separated NBSs (Figure 2d) that are conveniently represented by a dot-bracket notation (Figure 2c). Each NBS has been described by an RNA Sequence-Structure Pattern (RSSP), i.e., a pair composed of a string of bases (the sub-sequence corresponding to the NBS) and a string that represents the secondary structure in the dot-bracket notation (the NBS). In addition, a list of parameters is associated to each RSSP and composes the header line. The set of RSSPs makes up the Secondary Structure Descriptor (SSD) of the RNA sequence (Figure 2e).

$S S D_{-}$finder takes as input each match $m_{i}$ (with $1 \leq i \leq$ $n-1, n$ number of NBSs) between the reference and target, and returns groups of matches that may correspond to common structural motifs.

We consider the chains of matches $(C)$, as eligible chains if they:

1. consist of the largest number of consecutive NBSs; 2. exhibit similar relative distances between NBSs.

In particular, $S S D_{-}$finder is a dynamic programming algorithm that computes for each match $m_{i}$ only the

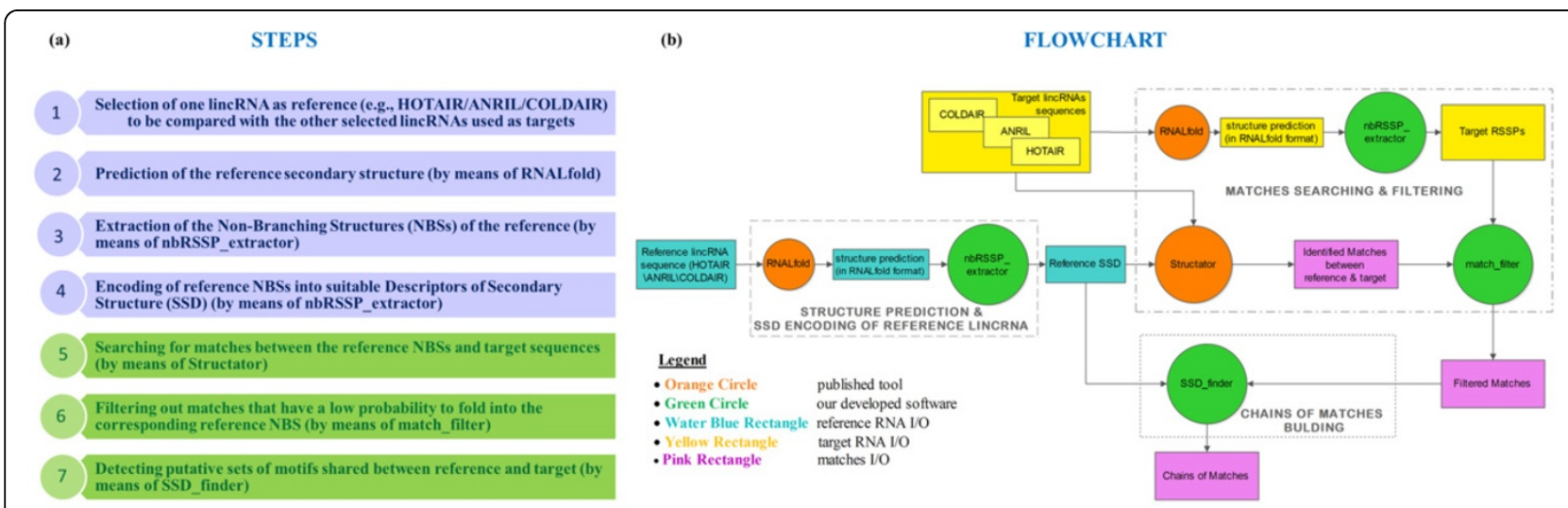

Figure 1 Pipeline steps and flowcharts of the whole procedure applied to lincRNAs. (a) The pipeline steps are explained and the two main parts of the procedure are highlighted: (1) the prediction and encoding of the lincRNA Secondary Structure Description (SSD) (blue part) and (2) the effective search engine for groups of matches (i.e., chains) common to the reference and target RNAs (green part). (b) The flowcharts of the experimental procedure is presented: given as input the reference lincRNA (HOTAIR or ANRIL or COLDAIR) and the target list composed of HOTAIR, ANRIL, and COLDAIR, the pipeline returns as output the potential structural motifs shared between reference and target. Legend: circles represent the software tools: the orange ones refer to two online available tools (i.e., RNALfold for prediction and Structator for the matches searching), the green ones refer to our developed software; rectangles represent software input and output (I/O), colored with water blue and yellow for what concerns reference and target, respectively; pink rectangles represent the final results after matching reference in the target. 
(a) RNA sequence

\section{AGCAACCUUGACUACCUAACGUUCCUAACAAGAGGAGCCACUAACAAGAGGGCACGAAGGGUAGCAAGGUCGUA}

(b) RNA secondary structure

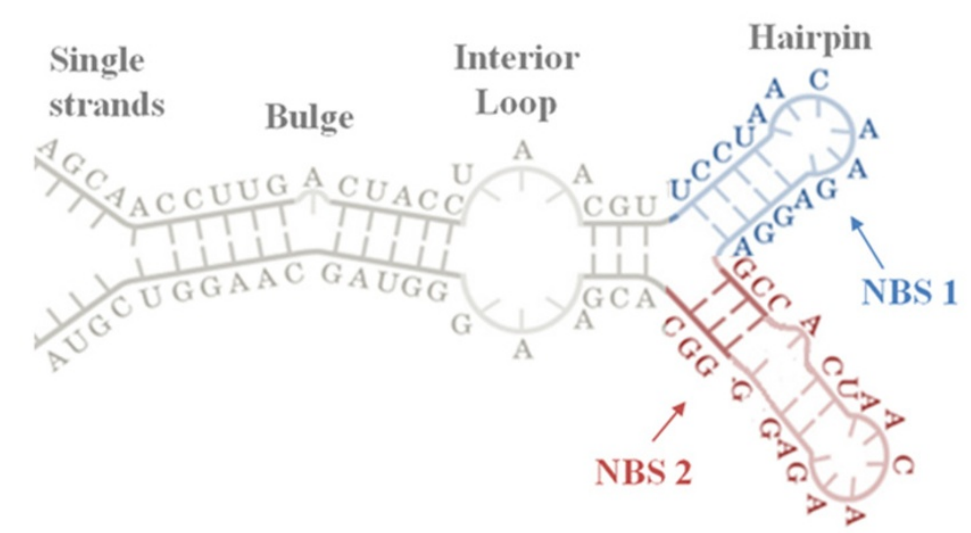

(c) Mapping of secondary structure in dot-bracket notation

RSSP1

RSSP2

AGCAACCUUGACUACCUAACGUUCCUAACAAGAGGA GCCACUAACAAGAGGGCACGAAGGGUAGCAAGGUCGUA

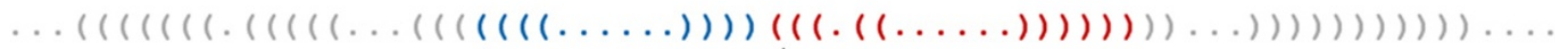
startpos=23 startpos $=37$

(d) Extraction of the Non-branching structures (NBSs)

NBS 2

(e) Encoding of the Non-branching structures (NBSs) Into Secondary Structure Descriptor

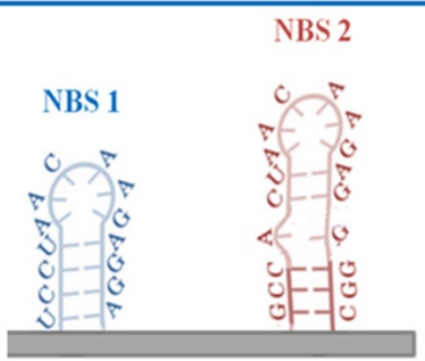

>RSSP1 | startpos $=23$
UCCUAACAAGAGGA
$((((\ldots \ldots)))$
>RSSP2 | startpos $=37$
GCCACUAACAAGAGGGC
$(((.((\ldots \ldots))))$

Figure 2 An example of the encoding of an RNA secondary structure into a Secondary Structure Descriptor (SSD). (a) RNA secondary structure representation with the two highlighted Non-Branching Structures (NBSs) (red one and blue one); (b) the extraction of the two NBSs; (c) mapping of the secondary structure in the dot-bracket notation (i.e., a 3-letter alphabet where dots represent unpaired bases, open-closed brackets "()" represent the paired bases) and the visualization of the two RSSPS that are a pair of the sub-sequence and the corresponding NBS; (d) the SSD composed of the two RSSPs.

highest score of the best chain ending with $m_{i}$ using the following score function:

$$
s c(C)=\sum_{i=1}^{n} P\left(m_{i}\right)+\sum_{i=1}^{n-1} Q\left(m_{i}, m_{i+1}\right)
$$

where:

- $P\left(m_{i}\right)$ is the weight of match $m_{i}$ taking into account its individual relevance (i.e., number of bases); 
- $Q\left(m_{i}, m_{i+1}\right)$ is a weight taking into account how much the pair $\left(m_{i}, m_{i+1}\right)$ in the target has positions consistent with the corresponding NBSs in the reference.

Therefore, chains with the highest scores are candidates to represent structural motifs shared between reference and target RNA.

Formally, let's define:

- $R$ the reference sequence;

- $T$ the target sequence;

- $S$ the list of NBSs extracted from the predict structure of $R$, that are sorted based on increasing starting positions;

- $s_{i}$ the $i$-th NBS in $S, \operatorname{pos}\left(s_{i}\right)$ its position in $R$ and len $\left(s_{i}\right)$ its length $(1 \leq i \leq n-1, n=|S|)$;

- $M$ the list of matches that are found in $T$ ordered in increasing sequence positions;

- $m_{i}$ the $i$-th match in $M, \operatorname{pos}\left(m_{i}\right)$ its position in $T$, len $\left(m_{i}\right)$ its length, and $\operatorname{nbs}\left(m_{i}\right)$ the NBS in $S$ which $m_{i}$ corresponds to;

- ind(.) an operator that starting from 1 returns the index of the argument (i.e., either a NBS in the list $S$, or a match in the list $M$ );

Specifically, matches of a list $M$ are pairs consisting of:

- index $i$ of $S$ of the matching NBS $s_{i}$;

- position $\operatorname{pos}\left(m_{i}\right)$ of matching subsequence of $T$ (i.e., a subsequence that can fold into $s_{i}$ based on the base pairing rules).

Note that $M$ is composed of all potential matches, including overlapping matches. Moreover, for what concerns the list $S$ of NBSs we have to distinguished two possible cases:

- case $S^{(1)}$ : the list $S$ is composed of only non-overlapped NBSs that form a unique prediction of the $R$ structure. Formally, $S$ contains only $s_{i}$ such that the $\operatorname{pos}\left(s_{i}\right)+\operatorname{len}\left(s_{i}\right) \leq \operatorname{pos}\left(s_{i+1}\right)$ condition is satisfied for any $i$;

- case $S^{(\mathrm{k})}$ : the list $S$ includes even overlapped NBSs that form $k$ possible alternative predictions of $R$ structure. Formally, $S$ contains $s_{i}$ such that may exist indices $i, j$ with $i<j$ implying $\operatorname{pos}\left(s_{i}\right)+\operatorname{len}\left(s_{i}\right)>\operatorname{pos}\left(s_{i+1}\right)$.

Note that case $S^{(1)}$ is the only option implemented in [36]. Furthermore, we remark that our nbRSSP_extractor can be switched between the two specific options to enable extracting either only the non-overlapped NBS from the RNALfold predictions (in the case $S^{(1)}$ ) or all the even overlapped NBSs (in the case $S^{(\mathrm{k})}$ ), according to the analyzed case study.

Finally, we define a chain $C=\left\{m_{j_{1}}, m_{j_{2}}, \ldots, m_{j_{n}}\right\}$ of matches in $M$ as a group of matches satisfying the following conditions $\forall i, 1 \leq i \leq n-1$ with $n=|\mathrm{M}|$ : (i) ind $\left(\operatorname{nbs}\left(m_{j_{i}}\right)\right)<$ ind $\left(\operatorname{nbs}\left(m_{j_{i+1}}\right)\right)$

(ii) $\operatorname{pos}\left(m_{j_{i}}\right)+$ length $\left(m_{j_{i}}\right) \leq \operatorname{pos}\left(m_{j_{i+1}}\right)$

In the following, we will denote the matches in a chain as $m_{1}, \ldots, m_{n}$ since the condition (ii) implies that $C$ is sorted according to increasing positions in $T$, which implies that $j_{i}<j_{i+1}, \forall_{i}, 1 \leq i \leq n-1$, and hence there are no ambiguities on match indices.

More details about the whole procedure are explained in specific sections of the additional file 2 (Supplementary_Material), in the additional file 3 (User_Guide), and additional file 4. (Figure S1).

\section{SSD_finder complexity}

$S S D \_$finder algorithm looks for the most meaningful chains of matches using the dynamic programming and regarding for all the matches $m_{i} \in M$ only the chains ending with $m_{i}$ that have the highest score. Let now consider $f=\operatorname{ind}\left(m_{i+1}\right)$ and $g=\operatorname{ind}\left(m_{i}\right)$ such that the condition (i) and (ii) are satisfied.

We use a score function $Q(f, g)$ that assigns a score to the pair of matches $\left(m_{i}, m_{i+1}\right)$. The complexity of our $S S D_{f}$ finder can be evaluated by counting the times the $Q$ function is called. The dynamic programming algorithm calls the $Q$ function once for any pair $(f, g)$ with $g<f$. Hence, the cost of the programming algorithm is:

$$
\sum_{f=1}^{n} \sum_{g=1}^{f-1} h(f, g)
$$

where $h(f, g)$ is the cost of the call to function $Q(f, g)$. In the case $S^{(1)}, h(f, g)$ is a constant for any $f, g$, leading to a total cost equal to $\frac{n(n-1)}{2}$. Therefore, the computational complexity of the $S S D_{-}$finder algorithm when we have only a unique prediction of $R$ is equal to $O\left(n^{2}\right)$.

In the case $S^{(\mathrm{k})}, h(f, g)$ accounts for the alternative perditions of the same structure of $R$. Firstly, we remark that $Q(f, g)$ weight $S$ how much the pair $(f, g)$ has positions consistent with the corresponding NBSs in $R$. Therefore, the $Q$ function depends on a distance $d(f, g)$ between $f$ and $g$ with $g<f$. We define the distance $d(f, g)$ as 1 plus the maximum number of non-overlapped NBSs $s_{k}$ such that $g<k<f$. Note that according to this definition, if there are no non-overlapped NBSs between $s_{f}$ and $s_{g} d(f, g)=1$.

To compute $d(f, g)$, the following algorithm can be used:

1 Initialize $l=f ; D=\{\}$;

2 Compute

$$
S=\left\{k \mid g<k<f \wedge \operatorname{pos}\left(s_{k}\right) \leq \operatorname{pos}\left(s_{l}\right) \wedge \operatorname{pos}\left(s_{g}\right)+\operatorname{len}\left(s_{g}\right) \leq \operatorname{pos}\left(s_{k}\right)\right\}
$$

3 If $S \neq\{\}$ compute 


$$
\begin{aligned}
& \quad \begin{array}{l}
j=\max (S) \\
D=D \cup j
\end{array} \\
& l=j ; \text { and repeat step } 2, \\
& \text { otherwise, } \\
& d(f, g)=1+|D| \text {; and stop. }
\end{aligned}
$$

Since the algorithm must consider all NBSs between $f$ and $g$, its complexity is $(f-g)$, and this is also the cost of function $Q(f, g)$. Hence, the complexity of dynamic programming algorithm is:

$$
\sum_{f=1}^{n} \sum_{g=1}^{f-1} f-g=\sum_{f=1}^{n} f(f-1)-\frac{f(f-1)}{2}=\frac{1}{2} \sum_{f=1}^{n}\left(f^{2}-f\right)
$$

that results equal to $\frac{n\left(n^{2}-1\right)}{6} \cong O\left(n^{3}\right)$ according to

the summation formulae of $\sum_{f=1}^{n} f^{2}=\frac{n(n+1)(2 n+1)}{6}$

and $\sum_{f=1}^{n} f=\frac{n(n+1)}{2}$. Therefore, the computational complexity of the $S S D_{-}$finder algorithm when we have more alternative predictions of $R$ is equal to $O\left(n^{3}\right)$.

\section{Validation procedure}

In the following, we define the experiments carried out to perform the $S S D_{-}$finder validation.

The validation procedure aims to test the results of our algorithm to identify members of RNA families obtained from the Rfam 11.0 database [38]. It can be summarized in the following steps: (i) we build up a target dataset composed of several Rfam families (i.e., more than 700 sequences belonging to different families); (ii) we select a whole sequence belonging to a given Rfam family as reference; (iii) we run the $S S D_{-}$finder obtaining the score of each sequence; (iv) we sort all the sequences of the target dataset in a decreasing order according to the returned score.

In greater details, among the reference Rfam families, we identify the following ones: (i) the Citrus tristeza virus replication signal family (RFAM Acc.:RF00193) composed of 44 sequences with an average length of 267 nucleotides (nt) (longest sequence length $=274 \mathrm{nt}$, shortest sequence length $=214 \mathrm{nt}$ ); (ii) the small ncRNAs OxyS family (RFAM Acc.: RF00035) composed of 299 sequences with an average length of $109 \mathrm{nt}$ (longest sequence length = $114 \mathrm{nt}$, shortest sequence length $=68 \mathrm{nt}$ ); (iii) the IncRNAs family HAR1A (RFAM Acc.: RF00635) composed of 66 sequences with an average length of $118 \mathrm{nt}$ (longest sequence length $=135 \mathrm{nt}$, shortest sequence length = $96 \mathrm{nt}$ ). Furthermore, the target dataset is composed of 723 sequences, including the abovementioned families and a subset of families randomly extracted from the Rfam 11.0 (http://rfam.sanger.ac.uk/) and RNAstrand v2.0 (http://www.rnasoft.ca/strand/) databases, whose shortest, longest, and average sequence length are equal to $43 \mathrm{nt}, 551 \mathrm{nt}$, and $201 \pm 8 \mathrm{nt}$, respectively.

\section{Results and discussion Validation}

To evaluate the robustness of $S S D_{-}$finder, we extensively test its performances in the identification of members of an RNA family. Specifically, the latter has been obtained from the online freely available Rfam 11.0 database [38], which consists of a curated collection of related RNAs. Note that the Rfam database selects only a short portion of the sequences which belongs to the Rfam families.

In the following, we explain the results of the experiments that we carried out to perform the $S S D_{-}$finder validation.

With reference to our validation procedure explained in the last subsection of Materials and Methods, in [36] we focused on the recognition of the consensus structure of some Rfam families (i.e., RF00193, RF00035, RF00635) for the selected short portion of these sequences. We observed that our SSD_finder was able to detect more than $80 \%$ of the members of families with high specificity. In Figure S2, additional file 5, we report the trend of the score computed by $S S D_{-}$finder with respect to the target sequences to be covered.

Here, we add noise in the input of SSD_finder, considering the whole original sequence for the member of the investigated family. In greater details, we test the $S S D_{-}$finder performances in the identification of a whole sequence randomly chosen from those belonging to the Rfam families. In fact, the Rfam families are composed of manually-curated fragments of RNA sequences. However, we aim to identify the whole sequence (reference RNA) as belonging to the right Rfam family among several fragments of sequences (target RNA). In this way, it should be more difficult to pick the right family to which the sequence belongs. However, we find that SSD_finder ranks the reference sequence in the top three of the score ordered list. Thus, our SSD_finder succeeds in finding the right family to which the whole sequence belongs even if the target dataset is only composed of sequence segments that made the detection harder.

More details about the score ranking and all the validation procedure are discussed in a specific section of the additional file 2 (Supplementary_Material) and in the additional file 6 (Figure S3).

We conclude that our algorithm is highly effective to discriminate the sequence belonging to the reference Rfam family.

\section{Prediction}

We study three lincRNAs, HOTAIR, ANRIL, and COLDAIR that constitute exemplar lincRNAs whose function seems to be related to their structure (Table 1). 
We apply both the SSD_finder flags, above referred as case $S^{(1)}$ and case $S^{(\mathrm{k})}$, for each combination of the three lincRNAs. The results are reported in Table 2 and Table 3, respectively. In greater details, in both Tables the score values computed by equation (1) and the length of the best chain of matches between reference and target lincRNA have been reported for each lincRNA.

In Table 2 we show performance of SSD_finder in case $S^{(1)}$, where only a unique prediction of the reference structure has been considered. This corresponds to the only case implemented in MONSTER v1.0. When each lincRNA is run against itself, the chain length found in the target is exactly equal to the number of RSSPs of the reference. To such a specific match MONSTER v1.1 assigns the highest score (bold numbers). We conclude that $S S D_{-}$finder succeeds to dredge the whole SSD of the reference lincRNA in the target lincRNAs list.

However, when each lincRNA is run against the others, SSD_finder works quite well. For example, HOTAIR (reference) versus ANRIL (target) show a shared chain of length 5 and score 7.24. It means that 5 non-branching structures are common between HOTAIR and ANRIL, building a common structural motif. Following the idea that a way of functioning of lincRNAs relies on their structures, this result could be a functional signature to infer a putative mechanism of action shared by HOTAIR and ANRIL.

In Table 3 we report the performances of SSD_finder on the lincRNA in case $S^{(\mathrm{k})}$, or when it takes into account all alternative structure predictions of the reference lincRNA. This can be clearly seen by the number of reference lincRNA RSSPs that is drastically higher than that of the corresponding field of Table 2. Indeed, the SSD of HOTAIR in Table 2 is composed of 67 nonoverlapped RSSPs that constitute a unique prediction of its structure, while the corresponding SSD in Table 3 is composed of 241 RSSPs, including the overlapping ones that represent the alternative predictions. Even here, $S S D_{-}$finder succeeds to identify the entire chain of lincRNA reference, providing different possible configurations of such a chain. In fact, since in this case even alternative NBSs of the reference lincRNA are taken into account, the chain lengths are always greater than the corresponding ones in Table 2 whose NBSs did not overlap. Furthermore, it found some chains of matches shared between different lincRNA (e.g., HOTAIR versus ANRIL show a common chain of 21 RSSPs and 38.5 score) that can suggest as abovementioned some putative common structural motifs.

Interestingly, looking at the list of common RSSPs between all the pairwise comparisons (e.g., HOTAIR and ANRIL; HOTAIR and COLDAIR; etc...), we have noticed the following meaningful characteristics (data not shown):

(i) 2 RSSPs of the chain shared between HOTAIR and ANRIL are also common between HOTAIR and COLDAIR, (ii) 4 RSSPs of the shared chain between ANRIL and HOTAIR are also among those ones that compose the shared chain between ANRIL and COLDAIR, and finally (iii) 3 RSSPs of the 7 ones that compose the shared chain between COLDAIR and HOTAIR are even among those ones of the COLDAIR and ANRIL shared chain. Therefore, we conclude that some structural motifs are shared among all the three lincRNAs, pointing to putative shared function of HOTAIR, ANRIL and COLDAIR.

Note that both Tables are not symmetrical for the exchange of the reference with target and viceversa and the results differ depending on which one is chosen as reference. This is due to the representation of the secondary structure to be searched for in the target sequence that is the result of a structure prediction and hence leads to some false positive and false negative values. Of course, the group of RSSPs that can be putative common structural motifs should be those ones found with a reference.

Finally, in order to give a statistically significance to our predictions, we computed 1000 shuffled versions of the three analyzed lincRNAs and we compared the score of the original comparisons with respect to those obtained by unrelated sequences. In this way, we are able to assign p-values to the scores of the original comparisons. In particular, we computed the zscore distribution.

$$
\text { (zscore }=\frac{\mathrm{x}-\mu}{\sigma} \text { with } \mu=\text { mean, } \sigma=\text { standard devia- }
$$

tion) of the highest scores of the unrelated sequences. Then, we located the original scores on this distribution and we found that they rely on the tails of the distribution corresponding to a p-value. The supplementary Figure S4, additional file 7 , sketches the computed

Table 2. Results of chain predictions about the HOTAIR, ANRIL and COLDAIR lincRNAs when a unique prediction for the reference lincRNA is considered (case $S^{(1)}$ )

\begin{tabular}{|c|c|c|c|c|c|c|}
\hline Target & & TAIR & & JRIL & & DAIR \\
\hline Reference (number of RSSPs) & Score & Chain length & Score & Chain length & Score & Chain length \\
\hline HOTAIR (67 RSSPS) & 224.5 & 67 & 7.24 & 5 & 6.80 & 5 \\
\hline ANRIL (96 RSSPS) & 4.78 & 4 & 351.7 & 96 & 5.98 & 4 \\
\hline COLDAIR (25 RSSPS) & 3.06 & 2 & 5.04 & 3 & 95.9 & 25 \\
\hline
\end{tabular}


Table 3. Results of chain predictions about the HOTAIR, ANRIL and COLDAIR lincRNAs when alternative predictions for reference lincRNA are considered (case $S^{(\mathbf{k})}$ )

\begin{tabular}{|c|c|c|c|c|c|c|}
\hline \multirow{2}{*}{$\begin{array}{l}\text { Target } \\
\text { Reference (number of RSSPs) }\end{array}$} & \multicolumn{2}{|c|}{ HOTAIR } & \multicolumn{2}{|c|}{ ANRIL } & \multicolumn{2}{|c|}{ COLDAIR } \\
\hline & Score & Chain length & Score & Chain length & Score & Chain length \\
\hline HOTAIR (241 RSSPs) & 271.9 & 74 & 38.5 & 21 & 18.6 & 10 \\
\hline ANRIL (335 RSSPS) & 29.4 & 18 & 412.4 & 111 & 18.1 & 10 \\
\hline COLDAIR (98 RSSPS) & 13.9 & 7 & 13.8 & 7 & 111.8 & 25 \\
\hline
\end{tabular}

zscore distributions and the zscore corresponding to our original score. We noticed that our original scores rely on the tail of the distribution corresponding to a p-value $\approx 0$.

\section{Conclusions}

We applied MONSTER v1.1 to search for the potentially shared structural motifs of three lincRNAs: HOTAIR, ANRIL, and COLDAIR. We found that SSD_finder has been resulted highly specific in recognizing the lincRNA selected as reference in the target list. Moreover, it predicted putative shared chains of matches between different lincRNAs that can be used to point to a common way of functioning. Finally, our method can aid to assign a putative function to an uncharacterized RNA relied on their secondary structures.

\section{Software availability}

The developed software package is available as supplementary file (zipped file named: "archive_updated.zip", additional file 1). Please refer even to the archive.zip provided as supplementary material of our work [36] to obtain the previous version of our procedure.

\section{Additional material}

Additional file 1: It contains the MONSTER_v1.1 software package with the updated version of SSD_finder, the file "INSTALL" that guides the package installation, the "data" and "example_data" folders which store all the needed files, including example input file and a script to run the whole procedure.

Additional file 2: It contains details about the MONSTER procedure, the validation procedure, and the prediction results.

Additional file 3: It contains a detailed guide for MONSTER setting up and additional advanced information about the package algorithms and their application.

Additional file 4: Figure S1 - Relevance of the $\mathrm{Q}$ term of score function (1). It contains the supplementary figure that depicts an example of the Relevance of the $Q$ term of the score function into chaining. An example of the chaining step (step 7) that shows the relevance of evaluating the distance among the RSSPs along with the number of RSSPs to select the best chain of matches.

Additional file 5: Figure S2 - Chain scores evaluated from SSD_finder. It contains the supplementary figure that depicts the chain scores evaluated from SSD_finder. Each panel represents the efficiency of our SSD_finder in the classification of the members of the four analyzed Rfam families (i.e., (a) RF00193, (b) RF00035, (c) RF00635, and (d) RF1975). The $y$ axes represent the score of our algorithm, evaluated as in equation (1) in our manuscript; the $\times$ axis represents the number of RNA sequences that constitute the database used as target in the chaining validation. This database includes the four selected families and a subset of families randomly extracted from the Rfam and RNAstrand databases (more than 700 sequences in total). In each case, the score computed by SSD_finder drastically decreases approaching to the number of sequences that corresponds to the number of the family members, allowing a clear identification of the exact number of detected members. Additional file 6: Figure S3 - Ranking results of the chain score of SSD_finder in the validation procedure. It contains the supplementary figure that depicts the rank results. Each panel represents the efficiency of our SSD_finder in identifying the right Rfam family in at least the three positions. (a) Table with the ranking results, whose first column represents the sequences used as target ( $1=$ fragment of sequence belonging to the right detected Rfam family; $0=0$ ther sequences that do not belong to the detected Rfam family). (b), (c) Bar plots that represent the graphical representation of the ranking results. The $y$ axes represent the normalized score of our algorithm, evaluated as in equation (1) in our manuscript; the $x$ axes represent the number of RNA sequences that constitute the database used as target in the chaining validation. This database includes the Rfam family to which the whole sequence used as reference belongs and a subset of families randomly extracted from the Rfam database (more than 700 sequences in total). The first group of plots (b) sketches the first seven ranking results, the second one (c) represents the whole set of sequences.

Additional file 7: Figure S4 - Distribution of the zscores. It contains the supplementary figure that depicts the zscore distributions. Each panel represents the score distribution of zscore using a shuffled version of the lincRNA joint with the score of our original comparison: (a) HOTAIR has been chosen as reference and the shuffled versions of ANRIL as target; (b) HOTAIR has been chosen as reference and the shuffled versions of COLDAIR as the target; (c) COLDAIR has been chosen as reference and the shuffled versions of ANRIL as target. It has been noticed how in all the cases our score is statistically significant with respect to the random distribution, yielding on the tails of distribution and corresponding to a $\mathrm{p}$-value $\approx 0$.

List of abbreviations

- InCRNA = long non-coding RNA;

- lincRNA = large intergenic non-coding RNA;

- NBS = Non-Branching Structure;

- RSSP = RNA Sequence Structure Pattern;

- SSD = Secondary Structure Descriptor.

\section{Competing interests}

The authors declare that they have no competing interests.

\section{Authors' contributions}

$\mathrm{Gl}$ and PP conceived the core idea of the research. Gl and GF developed and implemented algorithms. GF tested extensively the implementation, collected and analyzed experimental data. GF and GI made interpretation of data. All the authors wrote the paper. All the authors read and approved the final manuscript.

\section{Acknowledgements}

G.F. acknowledges financial support from The Epigenomics Flagship Project (Progetto Bandiera Epigenomica) EPIGEN funded by Italian Ministry of Education, University and Research (MIUR) and the National Research 
Council of Italy (CNR). The authors would like to thank Prof. Manuela Helmer Citterich for fresh biological insights and Dr. Paola Bertolazzi for inspiring discussions on the dynamic programming algorithms. A special thanks to the engineer Emilio Coppa for his advises on advanced bash-scripting.

\section{Declarations}

Publication of this article has been funded by the Institute for Systems Analysis and Computer Science "A. Ruberti" (IASI) of the italian National Research Council (CNR) and by The Epigenomics Flagship Project (Progetto Bandiera Epigenomica) EPIGEN funded by Italian Ministry of Education, University and Research (MIUR).

This article has been published as part of BMC Genomics Volume 16 Supplement 6, 2015: Proceedings of the Italian Society of Bioinformatics (BITS): Annual Meeting 2014: Genomics. The full contents of the supplement are available online at http://www.biomedcentral.com/bmcgenomics/ supplements/16/S6.

\section{Authors' details}

'Institute for System Analysis and Computer Science "Antonio Ruberti" (IASI), National Research Council (CNR), Via dei Taurini 19, 00185 Rome, Italy. ${ }^{2}$ Department of Computer, Control and Management Engineering (DIAG), Sapienza University, Via Ariosto 25, 00185 Rome, Italy. ${ }^{3}$ Centro integrato di ricerca, Università Campus Bio-medico di Roma, Via Alvaro del Portillo 21, 00128, Rome, Italy.

Published: 1 June 2015

\section{References}

1. Mattick JS: The central role of RNA in human development and cognition. Febs Letters 2011, 585:1600-1616.

2. Sumazin $P$, Yang $X$, Chiu HS, Chung WJ, Iver A, Llobet-Navas D, Rajbhandari P, Bansal M, Guarnieri P, Silva J, Califano A: An Extensive MicroRNA-Mediated Network of RNA- RNA Interactions Regulates Established Oncogenic Pathways in Glioblastoma. Cell 2011, 147:370-381.

3. Prensner JR, Chinnaiyan AM: The emergence of IncRNAs in cancer biology. Cancer Discov 2011, 1(5):391-407.

4. Mercer TR, Dinger M, Mattick JS: Long non-coding RNAs: insights into functions. Nature Reviews Genetics 2009, 10:155-159.

5. Clark MB, Mattick JS: Long noncoding RNAs in cell biology. Seminars in Cell \& Developmental Biology 2011, 22(4):366-376.

6. Tano K, Akimitsu N: Long non-coding RNAs in cancer progression. Frontiers in Genetics 2012, 3(219).

7. Cesana M, Cacchiarelli D, Legnini I, Santini T, Sthandier O, Chinappi M, Tramontano A, Bozzoni I: A Long Noncoding RNA Controls Muscle Differentiation by Functioning as a Competing Endogenous RNA. Cell 2011, 147(2):358-369.

8. Quereshi IA, Mattick JS, Mehler MF: Long non-coding RNAs in nervous system function and disease. Brain Res 2010, 1138C:20-35.

9. Saxena A, Carninci P: Long non-coding RNA modifies chromatin: epigenetic silencing by long non-coding RNAs. Bioessays 2011, 33(11):830-839.

10. Wang KC, Chang HY: Molecular mechanisms of long noncoding RNAs. Mol Cell 2011, 43:904-914.

11. Rinn JL, Kertesz M, Wang J, et al: Functional demarcation of active and silent chromatin domains in human HOX loci by noncoding RNAs. Cell 2007, 129:1311-23.

12. Khalii AM, Guttman M, Huarte M, Garber M, Raj A, Rivea Morales D, Thomas K, Presser A, Bernstein BE, van Oudenaarden A, Regev A, Lander ES, Rinn JL: Many human large intergenic noncoding RNAs associate with chromatin-modifying complexes and affect gene expression. Proc Natl Acad Sci 2009, 106(28):11667-11672.

13. Guttman M, Amit I, Garber M, French C, Lin MF, Feldser D, Lander ES: Chromatin signature reveals over a thousand highly conserved large non-coding RNAs in mammals. Nature 2009, 458:223-227.

14. Ulitsky I, Shkumatava A, Jan CH, Sive H, Bartel DP: Conserved Function of lincRNAs in Vertebrate Embryonic Development despite Rapid Sequence Evolution. Cell 2011, 147:1537-1550.

15. Cabili MN, Trapnell C, Goff L, Koziol M, Tazon-Vega B, Regev A, Rinn JL: Integrative annotation of human large intergenic noncoding RNAs reveals global properties and specific subclasses. Genes \& Development 2011, 25:1915-1927.
16. Nagano T, Fraser P: No-Nonsense Functions for Long Noncoding RNAs. Cell 2011, 145:178-181.

17. Tsai MC, Manor O, Wan Y, Mosammaparast N, Wang JK, Lan F, Shi Y, Segal E, Chang HY: Long Noncoding RNA as Modular Scaffold of Histone Modification Complexes. Science 2010, 329:689-693.

18. Chang JL, Rinn HY: Genome Regulation by Long NonCoding RNAs. Biochem Annual Review 2012, 81:145-166.

19. Mathews DH, Turner DH: Prediction of RNA secondary structure by free energy minimization. Current Opin in Structl Biol 2006, 16(3):270-278.

20. Woese CR, Pace NR: Probing RNA Structure, Function, and History by Comparative Analysis. The RNA World, Cold Spring Harbor Monograph Archive, Long Island, New York 1993, 24:91-117, http://rna.cshl.edu/content/ free/chapters/04_rna_world_2nd.pdf.

21. Zucker M, Stiegler P: Optimal computer folding of large RNA sequences using thermodynamics and auxiliary information. Nucleic Acid Res 1981, 9:133-148.

22. Hofacker I, Fontana W, Stadler PF, Bonhoeffer LS, Tacker M, Schuster P: Fast Folding and Comparison of RNA Secondary Structures. Monatshefte für Chemie 1994, 125:167-188.

23. Zakov S, Goldberg Y, Elhadad M, Ziv-Ukelson M: Rich parameterization improves RNA structure prediction. J of Comp Biol 2011, 18(11):1525-1542.

24. Bernhart SH, Hofacker IL, Will S, Gruber AR, Stadler PF: RNAalifold: improved consensus structure prediction for RNA alignments. BMC Bioinformatics 2008, 9(474).

25. Turner DH, Mathews DH: Dynalign: An algorithm for finding secondary structures common to two RNA sequences. J Mol Biol 2002, 317:191-203.

26. Touzet $H$, Perriquet O: CARNAC: folding families of related RNAs. Nucleic acids research 2004, 32:W142-W145.

27. Xia T, SantaLucia JJ, Burkard M, Kierzek R, Schroeder S, Jiao X, Cox C, Turner D: Thermodynamic parameters for an expanded nearestneighbor model for formation of RNA duplexes with Watson-Crick pairs. Biochemistry 1998, 37:14719-14735.

28. Mathews D, Sabina J, Zuker M, Turner D: Expanded sequence dependence of thermodynamic parameters provides improved prediction of RNA secondary structure. Mol Biol 1999, 288:911-940.

29. Ding Y, Chan C, Lawrence C: RNA secondary structure prediction by centroids in a Boltzmann weighted ensemble 2005, 11(8):1157-1166.

30. Ding $Y$, Lawrence C: A statistical sampling algorithm for RNA secondary structure prediction. Nucleic Acids Res 2003, 31(24):7280-7301.

31. Lu ZJ, Gloor JW, Mathews DH: Improved RNA secondary structure prediction by maximizing expected pair accuracy. RNA 2009, 15(10):1805-1813.

32. Reuter JS, Mathews DH: RNAstructure: software for RNA secondary structure prediction and analysis. BMC Bioinformatics 2010, 11(129):1471-2105.

33. Hofacker IL, Priwitzer B, Stadler PF: Prediction of Locally Stable RNA Secondary Structures for Genome-Wide Surveys. Bioinformatics 2004, 20(2):186-190.

34. Lange SJ, Maticzka D, Möhl M, Gagnon JN, Brown CM, Backofen R: Global or local? Predicting secondary structure and accessibility in mRNAs. Nucleic Acids Res 2012, 40(12):5215-5226.

35. Meyer F, Kurtz S, Backofen R, Will S, Beckstette M: Structator: fast indexbased search for RNA sequence-structure patterns. BMC Bioinformatics 2011, 12(1):214.

36. Fiscon $\mathrm{G}$, Paci $\mathrm{P}$, Colombo $\mathrm{T}$, lannello $\mathrm{G}$ : A new procedure to analyze RNA Non-branching Structures. BSP Current Bioinformatics 2015, 10(2).

37. Chen SJ, Dill KA: RNA folding energy landscapes. Proceedings of the National Academy of Sciences 2000, 97(2):646-651.

38. Gardner PP, Daub J, Tate J, Moore BL, Osuch IH, Griffiths-Jones S, Finn RD, Nawrocki EP, Kolbe DL, Eddy SR, et al: Rfam: Wikipedia, clans and the "decimal" release. Nucleic acids research 2011, 39(suppl 1):D141-D145.

39. Gupta RA, Shah N, Wang KC, Kim J, Horlings HM, Wong DJ, Tsai MC Hung T, Argani P, Rinn JL Wang Y, Brzoska P, Kong B, Li R, West RB, van de Vijver MJ, Sukumar S, Chang HY: Long noncoding RNA HOTAIR reprograms chromatin state to promote cancer metastasis. Nature 2011, 463(7291):1071-1076.

40. Heo JB, Sung S: Vernalization-Mediated Epigenetic Silencing by a Long Intronic Noncoding RNA. Science 2011, 331(6013):76-79.

41. Kotake Y, Nakagawa T, Kitagawa K, Suzuki S, Liu N, Kitagawa M, Xiong Y: Long non-coding RNA ANRIL is required for the PRC2 recruitment to 
and silencing of p15INK4B tumor suppressor gene. Oncogene 2011, 30(16):1956-1962.

doi:10.1186/1471-2164-16-S6-S1

Cite this article as: Fiscon et al:: MONSTER v1.1: a tool to extract and

search for RNA non-branching structures. BMC Genomics 2015

16(Suppl 6):S1.

Submit your next manuscript to BioMed Central and take full advantage of:

- Convenient online submission

- Thorough peer review

- No space constraints or color figure charges

- Immediate publication on acceptance

- Inclusion in PubMed, CAS, Scopus and Google Scholar

- Research which is freely available for redistribution

Submit your manuscript at 